Monica FIN*

Università di Padova

\title{
DA ZARA ALLA BRITISH LIBRARY: IL CURIOSO VIAGGIO DEL VOCABOLARIO DEI TRE NOBILISSIMI LINGUAGGI DI IVAN TANZLINGHER ZANOTTI
}

Parole chiave: Ivan Tanzlingher Zanotti, Vocabolario dei tre nobilissimi linguaggi, storia del manoscritto.

Il Vocabolario dei tre nobilissimi linguaggi, ${ }^{1}$ un voluminoso dizionario multilingue redatto nella seconda metà del Seicento dal canonico zaratino Ivan (Giovanni) Tanzlingher Zanotti (1651-1732), è indubbiamente uno dei prodotti più significativi della tradizione lessicografica croata. Con questa imponente opera, che nella sua versione "definitiva" comprende oltre 87.000 unità lessicali croate, 34.000 italiane e 21.000 latine, l'Autore intendeva dimostrare la vitalità della lingua croata (o, come si diceva al tempo, "illirica") in un'epoca di crisi e decadenza per le arti e la letteratura della Dalmazia.

Malgrado il suo indiscusso valore, il Vocabolario di Tanzlingher è rimasto a lungo inaccessibile al pubblico e alla critica poiché, di fatto, non fu mai dato alle stampe. Lo stesso Tanzlingher ne curò diverse redazioni, cui sembrano corrispondere altrettante copie riemerse solamente a partire dalla metà del sec. XX ed oggi conservate a Zagabria, Padova, Londra e Zara. Grazie a questi ritrovamenti il Vocabolario dei tre nobilissimi linguaggi è divenuto un punto fermo della tradizione lessicografica croata, oltre ad essere stato oggetto di numerosi contributi scientifici.

*monica.fin@unipd.it

${ }^{1}$ Il titolo completo dell'opera è Vocabolario dei tre nobilissimi linguaggi, italiano illirico e latino [...] raccolto dal Molto Reverendo Signor D. Giovanni Tanzlingher, Dottor, e Canonico di Zara. 
Il presente lavoro costituisce la fase preliminare di una ricerca dedicata alla copia del Vocabolario oggi conservata presso la British Library di Londra. I contributi finora prodotti sull'argomento - pochi, per la verità - si sono concentrati prevalentemente sugli aspetti formali, grafici e lessicografici del manoscritto. ${ }^{2}$ Minor attenzione, invece, è stata dedicata alla sua genesi e al percorso che da Zara, dove (pare) fu redatto, lo portò fin sugli scaffali di una delle maggiori biblioteche del mondo. Nei prossimi paragrafi ci concentreremo proprio su questi due punti.

\section{Il Vocabolario dei tre nobilissimi linguaggi}

Le quattro copie del Vocabolario di Tanzlingher ad oggi note sono profondamente diverse fra loro, al punto da venire generalmente trattate come diverse "versioni", o addirittura "edizioni" dell'opera. Per maggior chiarezza ne elencheremo brevemente le caratteristiche, seguendo l'ordine cronologico di ritrovamento dei manoscritti. ${ }^{3}$

La prima copia del Vocabolario è conservata a Zagabria, presso 1'Archivio dell'Accademia croata delle Scienze e delle Arti, dove giunse nel 1898. Il manoscritto è costituito da un unico volume di 164 fogli, redatti in italiano, croato e latino. Fino a tempi molto recenti si riteneva che la copia zagabrese, che porta la data 1679, contenesse la prima edizione (forse autografa) del Vocabolario di Tanzlingher. ${ }^{4}$

La seconda copia ad essere stata scoperta e descritta dagli studiosi si trova a Padova, presso la Biblioteca del Dipartimento di Studi Linguistici e Letterari. ${ }^{5} \mathrm{Il}$ manoscritto è composto da due grandi volumi in folio $(\mathrm{cm} .32 \mathrm{x}$

${ }^{2}$ Il primo (e ad oggi l'unico) studio monografico dedicato alla copia di Londra è quello pubblicato da V. Bockholt, Z. Mestrović, N. Vajs, Nepoznati dvojezični rječnik Ivana Tanclinghera Zanottija, «Filologija», knj. 36-37 (2001), pp. 45-59.

${ }^{3}$ Per una descrizione dettagliata delle quattro copie ad oggi note del Vocabolario di Tanzlingher si rimanda a: R. Benacchio, H. Steenwisk, Per un'edizione on line del Vocabolario di tre nobilissimi linguaggi, italiano, illirico e latino di Giovanni Tanzlingher Zanotti (1699-1704), in Uomini, opere e idee tra Occidente europeo e mondo slavo. Scritti offerti a Marialuisa Ferrazzi, a cura di A. Mingati, D. Cavaion, C. Criveller, Trento, 2011, pp. 41-56; N. VAJS, Još jedan rukopisni rječnik Ivana Tanzlinghera Zanottija, «Rasprave: C̆asopis Instituta za hrvatski jezik i jezikoslovlje», 37/1 (2011), pp. 199-260.

${ }^{4}$ T. MATić, Prva redakcija Tanclingherova riječnika, «Rad JAZU», 293 (1953), pp. 253-279: 255.

${ }^{5}$ Il manoscritto fu acquistato dall'Istituto di Filologia slava di Padova grazie ai fondi della Dotazione ministeriale per l'a.a. 1952/1953. La copia padovana del Vocabolario fu presentata alla comunità scientifica nel 1959, grazie ad un esauriente contributo di Jolanda 
45) per un totale di 1316 pagine. Il primo volume si apre con una Prefazione in lingua croata datata 22 maggio 1704; l'intestazione della pagina con cui ha inizio il vocabolario vero e proprio, invece, porta la data 1699. Anche in questo caso si tratta di un dizionario trilingue, stilato in italiano, croato e latino. Entrambi i volumi recano l'Ex Libris dello storico zaratino Giuseppe Praga. ${ }^{6}$ La copia padovana, contenutisticamente più ricca rispetto alle altre, viene considerata il frutto maturo dell'opera lessicografica di Tanzlingher e costituisce (probabilmente) la redazione "definitiva" del suo Vocabolario. ${ }^{7}$

La terza copia del Vocabolario è conservata presso la British Library (coll. MS 10.360). Si tratta di un manoscritto in volume unico di 258 fogli (cm. 20 x 28), recante il titolo Vocabulario Italiano ed Ilirico. Raccolto da Don Giovanni Tanzlingher. Dottore e Canonico di Zara l'anno 1699. La copia londinese si differenzia dalle altre per alcune importanti caratteristiche formali: in primo luogo, e come suggerito dal titolo stesso, è l'unica ad essere redatta in due sole lingue, italiano e croato, ${ }^{8}$ mentre manca del tutto la parte latina; in secondo luogo, è l'unica a essere priva di prefazione e/o

Marchiori. Cfr. J. MARChIORI, Note al Vocabolario dei tre nobilissimi linguaggi italiano, illirico e latino del 1704 di Giovanni Tanzlingher Zanotti, «Atti e memorie dell'Accademia Patavina di Scienze, Lettere ed Arti», Parte 3, Memorie della Classe di Scienze Morali, Lettere ed Arti, Vol. LXXII (1959), pp. 19-50.

${ }^{6}$ La presenza nella copia padovana dell'Ex libris dello storico zaratino ha permesso di ricostruire il percorso di questo prezioso manoscritto. Dopo essere rimasto a lungo in possesso della famiglia Filippi di Zara, esso fu acquistato da Praga (probabilmente) in occasione di un'asta antiquaria. Lo stesso Praga spedì i due volumi in Italia nell'estate del 1943, assieme ai pezzi più preziosi della sua biblioteca privata. Nel 1952, infine, la copia del Vocabolario giunse a Padova grazie all'iniziativa di Arturo Cronia, che in quegli anni, in qualità di Direttore dell'Istituto di Filologia slava, si occupava anche della gestione della biblioteca. Per approfondimenti cfr. G. FerRARI-CuPILl, Della vita e degli scritti di Giovanni Tanzlingher Zanotti Canonico Zaratino, «Annuario dalmatico», II (1861), pp. 77-103; M. FIN, Arturo Cronia e la Biblioteca di Slavistica a Padova, in R. Benacchio, M. Fin, Arturo Cronia. L'eredità di un Maestro a cinquant'anni dalla scomparsa, Padova, 2019, pp. 93-116.

${ }^{7}$ Vorrei qui ricordare la ricerca condotta sulla copia padovana da un gruppo di studiosi dell'Università di Padova in collaborazione con i colleghi dell'Institut za hrvatski jezik i jezikoslovlje di Zagabria, conclusasi con la pubblicazione on-line del Vocabolario_(cfr. http://tanzlingher.filosofia.sns.it). Per una descrizione delle attività svolte nell'ambito del progetto cfr. R. Benacchio, H. Steenwisk, Ž. Jozić, N. Vajs Vinja, Digitalna obradba rukopisnoga rječnika Vocabolario di tre nobilissimi linguaggi, italiano, illirico, e latino Ivana Tanzlinghera Zanottija (1651-1732), «Filologija», 58 (2012), pp. 19-38.

${ }^{8} \mathrm{Nel}$ discutere la percezione che Tanzlingher aveva della lingua croata, Bockholt, Meštrović e Vajs puntualizzano come l'autore, originario di Zara e dunque parlante čakavo, abbia introdotto nel suo dizionario numerosi termini štokavi e kajkavi («on kao rođeni čakavac u Zadru u svoj rječnik svjesno unosi brojne štokavizme (turcizme) i kajkavizme 
postfazione; è inoltre l'unica ad essere corredata dall'appendice intitolata Raccolta d'alcuni termini militari che s'attrovano sparsi nel Libro Maresciallo; infine, ad un confronto con le altre copie ad oggi note, quella di Londra si distingue per la sua «eccezionale correttezza linguistica». ${ }^{9}$

La quarta copia del Vocabolario, l'ultima in ordine di tempo a essere stata rinvenuta, è conservata presso la Biblioteca capitolare di S. Anastasia a Zara, dove è stata ritrovata nel 2009. Il manoscritto, redatto in italiano, croato e latino, è costituito da un volume unico di 159 fogli privo di prefazione e di data. ${ }^{10}$ Malgrado questa lacuna, studi recenti hanno dimostrato che la copia di Zara contiene la prima stesura del dizionario, probabilmente prodotta dal Tanzlingher negli anni giovanili. ${ }^{11}$

\section{La copia di Londra e i suoi "custodi"}

Dopo aver fatto il punto sulle diverse redazioni del Vocabolario, passiamo ora ad esaminare $i$ dati in nostro possesso in merito alla provenienza della copia conservata presso la British Library. ${ }^{12}$

Da una nota inserita nel secondo foglio di guardia apprendiamo che il manoscritto fu acquistato dalla British Museum Library durante un'asta tenutasi nel febbraio $1836,{ }^{13}$ come parte della collezione privata del noto

[...] navodi potvrde iz sva tri narječa»; V. Bocкholt, Z. Mestrović, N. VAjs, Nepoznati dvojezični rječnik Ivana Tanclinghera Zanottija, cit., p. 50).

${ }^{9}$ R. Benacchio, H. Steenwisk, Per un'edizione on line del Vocabolario di tre nobilissimi linguaggi, italiano, illirico e latino di Giovanni Tanzlingher Zanotti (16991704), cit., p. 44.

${ }^{10}$ Per una descrizione puntuale della copia di Zara cfr. N. VAJS, Još jedan rukopisni rječnik Ivana Tanzlinghera Zanottija, cit., pp. 224-228.

${ }^{11}$ R. Benacchio, H. SteEnwiJk, La Crusca come fonte lessicografica in area dalmatocroata: la copia padovana del Vocabolario dei tre nobilissimi linguaggi di G. TanzlingherZanotti (1651-1732), in Il mondo slavo e l'Europa, a cura di C. Bragone e M. Bidovec, Firenze, [Biblioteca di Studi slavistici, 43], 2019, pp. 25-34.

${ }^{12}$ La presenza del manoscritto presso la British Library fu segnalata da V. MARKotić, Manuscripts referring to Croatia in the British Museum in London, «Radovi hrvatskoga povijesnog instituta u Rimu», 3-4 (1971), pp. 263-292: 269.

${ }^{13}$ La nota recita «Purchased Feb. 1836. Heber's Sale. Lot 1679» (V. Bockholt, Z. Mestrović, N. Vass, Nepoznati dvojezični rječnik Ivana Tanclinghera Zanottija, cit., p. 46). Sulla base dei dati forniti da S. De Ricci possiamo ipotizzare che il manoscritto sia stato acquistato dal British Museum durante la vendita tenuta dalla casa d'aste londinese Evans il 10 Febbraio 1836 (S. DE Ricci, English Collectors of Books and Manuscripts, Cambridge, 1930, p. 103, nota 1). 
linguista e bibliofilo inglese Richard Heber (1773-1833). ${ }^{14} \mathrm{Il}$ primo foglio di guardia, invece, reca l'Ex Libris di Frederick North (1766-1827), V conte di Guilford, eccentrico e facoltoso lord inglese che di Richard Heber fu buon amico. Proprio su Guilford vale la pena di concentrarsi poiché, di fatto, si tratta del primo proprietario "attestato" del manoscritto di Londra. ${ }^{15}$

Nato in una famiglia dell'alta aristocrazia inglese (il padre, suo omonimo, fu Primo Ministro durante il regno di Giorgio III), Frederick North viene descritto dai suoi contemporanei come un uomo dai modi amichevoli, un abile diplomatico e un eccellente conversatore. Ricevette la classica educazione che si confaceva ad un gentiluomo inglese: dopo aver frequentato l'esclusivo collegio di Eton, studiò lingue e letterature classiche ad Oxford; scriveva in modo impeccabile sia in latino che in greco classico e parlava fluentemente francese, tedesco, spagnolo, italiano e russo. Durante gli studi universitari sviluppò un profondo interesse, durato poi tutta la vita, per la cultura ellenica: una passione, questa, che nel 1792 lo portò a convertirsi all'ortodossia.

Dopo aver lasciato Oxford nel 1793, il giovane North iniziò il suo Grand Tour. Partendo dalla Spagna, viaggiò per tutto il bacino del Mediterraneo, visitando le Isole Ionie e le coste orientali dell'Adriatico, passando per Smirne, Cipro, Alessandria, Gerusalemme, Costantinopoli e Dubrovnik. Al suo ritorno in Inghilterra prese il posto del padre, nel frattempo scomparso, come rappresentante della famiglia in parlamento. Ben presto il governo scorse in lui un brillante amministratore e prese ad affidargli incarichi sempre più importanti: North divenne così il primo governatore britannico di Ceylon, dove fu di stanza tra il 1798 e il 1805, e dopo il trattato di Vienna, con cui venne confermato il protettorato britannico sulle Isole Ionie, fu inviato a Corfù con l'incarico di riformare il sistema scolastico dell'isola.

${ }^{14}$ Heber soleva dire che «No gentleman can be without three copies of a book, one for show, one for use and one for borrowers». La sua biblioteca privata, fra le più ricche nell'Inghilterra dell'epoca, comprendeva oltre 200.000 volumi, molti dei quali preziosissimi. Dopo la sua morte essa fu smembrata durante 16 vendite, svoltesi fra il 1834 e il 1837 a Londra, Parigi e Gand. A dividersi i 1717 manoscritti che facevano parte della collezione furono il British Museum, la Bodleian Library di Oxford e il bibliofilo Sir Thomas Philipps (S. De Ricci, English Collectors of Books and Manuscripts, cit., p. 102-104).

${ }^{15}$ Per un profilo biografico di Frederick North si rimanda alla voce dedicata in Oxford Dictionary of National Biography (https://www.oxforddnb.com/). Inoltre, si vedano: M. PARTRIDGE, Jernej Kopitar and Frederick North, 5th Earl of Guilford, Kopitarjev zbornik, Ljubljana, 1996, pp. 277-284; K. WARE, The Fifth Earl of Guilford (1766-1827) and his Secret Conversion to the Orthodox Church, in Anglicanism and Orthodoxy 300 Years after the 'Greek College' in Oxford, a cura di P.M. Doll, Oxford-Bern 2006, pp. 289-326. 
Nel 1817, alla morte del fratello maggiore Francis, Frederick North fu creato V conte di Guilford. Il prestigio e le ingenti ricchezze garantite dalla sua posizione gli permisero di realizzare molti progetti vicini al suo cuore, tutti connessi con la sua passione per il mondo greco. Già durante il suo primo soggiorno sulle Isole Ionie, nel 1792, egli si era detto sconvolto dall'orribile patois parlato dagli isolani. ${ }^{16}$ Proprio come Ivan Tanzlingher, dunque, che credeva così tanto nel valore e nella ricchezza della lingua croata da dedicare buona parte della sua vita adulta alla compilazione del Vocabolario, Guilford, da convinto filellenico, investì tempo e denaro per salvaguardare e promuovere la lingua e la cultura greca. Insieme al conte Giovanni Capodistria, suo amico e sodale, concepì il progetto per la creazione di un'università con sede nelle Isole Ionie. Fu così che nel maggio del 1824 nacque l'Accademia Ionica, con sede a Corfù, la prima università dell'orbe greco che, nei piani del suo fondatore, non avrebbe avuto nulla da invidiare agli atenei di Oxford e Cambridge. L'Accademia, che utilizzava il greco moderno come lingua di insegnamento e comprendeva quattro facoltà (teologia, diritto, medicina e filosofia), conobbe un rapido sviluppo fino al 1827, anno in cui, del tutto improvvisamente, Guilford venne a mancare. Orfana del suo fondatore, della sua influenza personale e (soprattutto) del suo sostegno finanziario, l'Accademia declinò rapidamente e finì col chiudere nel 1864, complice l'annessione delle Isole Ionie alla Grecia. ${ }^{17}$

Durante i suoi lunghi viaggi, che lo portarono ad attraversare l'Europa intera, dalla Spagna alla Russia, Guilford fece la conoscenza di alcuni fra i più importanti linguisti e scrittori di lingua slava dell'epoca, con cui mantenne rapporti molto stretti. ${ }^{18}$ Fu buon amico di Jernej Kopitar, ${ }^{19}$ Josef Dobrovský, Luka Stulić e Pavle Solarić, per citarne solo alcuni; degno di nota è anche il suo rapporto con Francesco Maria Appendini, che lo

${ }^{16}$ M. PARTRIDGE, Jernej Kopitar and Frederick North, 5th Earl of Guilford, cit., p. 278.

${ }^{17}$ Per una storia dell'Accademia Ionica cfr. G.P. Henderson, The Ionian Academy, Glasgow, 1988.

${ }^{18}$ Malgrado i suoi stretti rapporti col mondo slavo, la figura di Guilford rimane poco nota presso gli studiosi serbi e croati. In lingua serba l'unico contributo a lui dedicato porta la firma di Marta Frajnd (M. Frajnd, Luka Stulić i Lord Nort-prilozi poznavanju veza Dubrovnika i Engleske u XIX veku, «Prilozi za KJIF», 77 (2011), pp. 105-114). In lingua inglese, invece, ricordiamo i lavori di Monica Partridge, secondo cui Guilford fu «the first true English Slavist» (M. PARTRIDGE, Jernej Kopitar and Frederick North, 5th Earl of Guilford, cit., p. 277).

${ }^{19}$ Kopitar lo definì «der Kenner und Freund der Slavischen Sprachen» (M. PARTRIDGE, An English Eccentric and Some Slavs and Slavists, «Wiener Slavistisches Jahrbuch», 21 (1975), 202-213: 202). 
stimava molto per le sue conoscenze linguistiche. ${ }^{20}$ Durante i suoi soggiorni in Dalmazia, in special modo a Dubrovnik $(1819,1819,1824)$ e a Zara, Guilford sviluppò un profondo interesse per la lingua croata, che pure lui chiamava «la langue illyrienne», al punto da iniziare a prendere lezioni private. ${ }^{21}$ Secondo la studiosa inglese Monica Partridge, a motivare questa scelta furono soprattutto finalità pratiche: egli intendeva imparare la lingua "illirica" per poter riconoscere gli elementi allogeni penetrati nella parlata delle Isole Ionie, che secondo lui doveva essere purificata. ${ }^{22} \mathrm{Si}$ tratta di un punto importante, su cui ritorneremo.

Oltre che per il suo profilo di uomo politico e mecenate, Guilford è ben noto agli studiosi britannici anche come collezionista e bibliofilo, in virtù della sua invidiabile raccolta privata di libri e manoscritti, alcuni particolarmente preziosi. ${ }^{23}$ L'interesse di Guilford per le lingue slave è ben riflesso anche nella sua biblioteca, valutata come la più grande raccolta privata di manoscritti e libri slavi ad essere mai stata assemblata in territorio inglese ${ }^{24}$ che comprendeva numerosi volumi in slavo ecclesiastico, russo, serbo e croato; la copia di Londra del Vocabolario di Tanzlingher faceva parte di questa collezione. ${ }^{25}$ Dopo la morte di Guilford, la biblioteca venne

${ }^{20}$ Appendini traccia un breve ricordo di Guilford nella Prefazione al De vita et scripta Bernardi Zamagnae (Zara 1830). Secondo Monica Partridge, c'è motivo di credere che Guilford fosse stato invitato ad intervenire ai lavori della Commissione ortografica, riunitasi a Zara nel 1820, e di cui Appendini, per l'appunto, era presidente (M. PARTRIDGE, The First Practical Grammar and Reader of Illyrian, Savremena slavistika, 20, Zagreb, 1996, 497-502: 502)

${ }^{21}$ Non è dato sapere con certezza quando Guilford abbia iniziato a prendere lezioni di lingua croata; sappiamo però che fra i suoi insegnanti vi fu anche Pavel Đuračić, nativo di Stagno e vicino alla cerchia dei latinisti ragusei, che in una lettera destinata al lord inglese si definisce «maître de la langue Illirique» (M. PARTRIDGE, An English Eccentric and Some Slavs and Slavists, cit., p. 205). I due si erano conosciuti ad Atene al principio degli anni '20, quando Đuračić si trovava in città come agente diplomatico. Oltre al croato, Guilford studiò anche il russo e la grammatica dello slavo ecclesiastico con Pavle Solarić, come testimonia una lettera di Jernej Kopitar dell'aprile 1816 in cui si legge: «Lord North lernt von Solarich in Venedig Slavisch» (M. PARTRIDGE, Jernej Kopitar and Frederick North, 5th Earl of Guilford, cit., p. 280).

${ }^{22}$ M. PARTRIDGe, The First Practical Grammar and Reader of Illyrian, cit., p. 498.

${ }^{23}$ Secondo le testimonianze dei contemporanei, Guilford considerava la sua biblioteca «the favourite child of my old age» e soleva dire: «If I were not the Earl of Guilford I would have liked to be a librarian» (K. WARE, The fifth earl of Guilford (1766-1827) and his secret conversion to the Orthodox church, cit., p. 315).

${ }^{24}$ M. PARTRIDGE, Jernej Kopitar and Frederick North, 5th Earl of Guilford, cit., p. 278.

${ }^{25}$ Il volume più prezioso fra quelli in lingue slave era senza dubbio il messale glagolitico noto come "Messale di New York", risalente all'inizio del XV secolo e 
smembrata e venduta all'asta a Londra, fra il 1828 e il 1835: durante le varie sedute furono battuti 3.000 manoscritti e 15.000 volumi a stampa. ${ }^{26}$ Probabilmente fu proprio allora che Richard Heber acquistò il manoscritto del Vocabolario. ${ }^{27}$

\section{Un dizionario e una grammatica}

Come già ricordato, i pochi studi finora condotti sulla copia londinese del Vocabolario di Tanzlingher si concentrano sulle sue caratteristiche formali, grafiche e contenutistiche. Per quanto riguarda la datazione del manoscritto, ad esempio, finora si è fatto fede alla data che compare nel titolo dello stesso, ossia l'anno 1699. Si è così ipotizzato che il dizionario sia stato compilato alla fine del XVII secolo, probabilmente sulla base della copia padovana, che riporta la stessa data e comprende quasi tutti i termini italiani e croati riscontrabili nella copia di Londra, con poche eccezioni. ${ }^{28}$ Ma se non fosse proprio così?

attualmente conservato presso la Morgan Library (cfr. The New York missal: an early 15th-century Croato-glagolitic manuscript, a cura di H. Birnbaum e P. Rehder, München/ Zagreb, 1977).

${ }^{26}$ Per un catalogo dei manoscritti appartenuti a Guilford cfr. Catalogue of the extraordinary, curious, and extensive collection of manuscripts of the Late Earl of Guilford ... sold by auction, by Mr. Evans, at his house, No. 93, Pall Mall, on Wednesday, December 8, and four following Days, Sunday excepted, [London] 1830. La maggior parte dei manoscritti (circa 700 pezzi) fu acquistata dal British Museum e si trova tutt'oggi presso la British Library. Nel 2006 la British Library ha lanciato un progetto volto a produrre un catalogo dei manoscritti provenienti dalla collezione Guilford. Per maggiori informazioni cfr. http://vll-minos.bl.uk/reshelp/findhelprestype/manuscripts/guilford/index.html.

${ }^{27}$ Più difficile è ipotizzare che Heber abbia acquistato il manoscritto da Guilford stesso, malgrado il rapporto di amicizia che li legava. Nel suo testamento, infatti, Lord North diede disposizioni affinché la sua biblioteca privata rimanesse presso l'Accademia Ionica, a condizione che il governo locale garantisse un'ingente donazione annuale all'università. Tuttavia, il suo erede, Lord Sheffield, sfruttò il mancato rispetto di questa clausola come pretesto per esigere la restituzione dell'intera collezione, che fu messa all'asta e venduta (K. WARE, The fifth earl of Guilford (1766-1827) and his secret conversion to the Orthodox church, cit., p. 323-324).

${ }^{28}$ Come nota Nada Vajs, «Broj hrvatskih istovrijednica u padovanskom i u londonskom rječniku je uglavnom podudaran, dakle mnogo bogatiji od istovrijednica u zagrebačkom i zadarskom, zbog maloga obima ovih potonjih rječnika. Unatoč podudarnosti hrvatskih istovrijednica u padovanskom (P) i u londonskom (L) rječniku, zamjećuju se razlike u odabiru talijanskih natuknica kod kojih se donose hrvatski ekvivalenti» (N. VAJs, Još jedan rukopisni rječnik Ivana Tanzlinghera Zanottija, cit., p. 45). 
$\mathrm{Ci}$ sono infatti altre caratteristiche importanti che sono tipiche solo della copia di Londra e che meritano di essere prese in considerazione. Alcune le abbiamo già menzionate: la mancanza della parte latina costituisce certamente la differenza più evidente, ma altrettanto evidenti sono alcune peculiarità nell'ortografia della copia londinese, molto più vicina a quella del croato moderno rispetto agli altri tre manoscritti. Infine, ad un confronto con le altre copie del Vocabolario, quella di Londra si distingue per la sua particolare precisione linguistica. Tutti questi elementi ci inducono a pensare che questo manoscritto potrebbe di fatto contenere una versione/edizione successiva dell'opera di Tanzlingher, sicuramente più tarda di quel 1699 che compare nel titolo.

Si possono quindi formulare delle ipotesi in merito alla genesi stessa del manoscritto, ipotesi che coinvolgono lo stesso Guilford e i suoi contatti nel mondo slavo: d'altra parte è indubbio che, qualora ne fosse sorto il bisogno, fra i numerosi conoscenti del nostro Lord (e penso soprattutto ai suoi sodali zaratini) vi era sicuramente qualcuno in grado di produrre una copia ad hoc dell'opera di Tanzlingher. Alcuni materiali conservati negli archivi inglesi e serbi sembrano puntare in questa direzione.

Da alcuni documenti dell'epoca, infatti, apprendiamo che Guilford aveva in progetto di creare una scuola di studi slavi e una stamperia dotata di caratteri cirillici all'interno dell'Accademia Ionica. Sfruttando la sua vasta rete di conoscenze egli si informò presso alcuni amici in merito alla disponibilità di insegnanti e libri per poter avviare i corsi. Kopitar, ad esempio, gli consigliò di arruolare niente meno che Vuk Karadžić come docente per la nuova cattedra di lingua slava, presentandolo come segue:

Non seulement il sait parfaitement le Slavon ancien, le Serbe moderne, a voyagé en Russie \& en Allemagne, a une réputation litéraire bien meritée par ses ouvrages [...] Il connait la Serbe moderne mieux que tout autre personne ... Du reste il est a l'age de 35 ans, bien pourtant, zéleux, a la meilleure tête illirique que j'ai rencontré, \& par conséquent d'un caractère doux, droit \& ferme. [...] Si je pouvais espérer que nous mêmes ferons quelque chose pur les Serbiens, je ne céderais cet excellent savant à personne. Cela n'étant pas, j'aime mieux le céder à l'occident qu'à l'Orient. ${ }^{29}$

${ }^{29}$ Nella stessa lettera Kopitar non manca di menzionare la malattia che affliggeva Vuk, costringendolo ad utilizzare una protesi di legno: «Il est vrai qu'il a une jambe de bois comme un officier estropié: mais qui sait, si le climat de Corfou ne lui dégèlera la concrétion au genou gauche, qui l'empêche de manier le pied gauche comme il fait du droit» (M. PARTRIDGe, Jernej Kopitar and Frederick North, 5th Earl of Guilford, cit., p. 282). 
Per quanto riguarda invece la stamperia slava, la difficoltà maggiore riguardava il reperimento dei caratteri di stampa. Oltre ad interpellare il solito Kopitar, in questo caso Guilford coinvolse anche Pavle Solarić: del resto, nessuno meglio di lui, che aveva lavorato per anni come revisore e correttore di bozze presso la casa editrice Teodosio di Venezia, poteva sperare di aver fortuna in questa missione. ${ }^{30}$

Nel corso degli anni, Guilford si avvalse dell'aiuto di alcuni intermediari per mettere insieme la sua immensa raccolta di libri e manoscritti: per i libri serbi si appoggiava a Kopitar e Solarić, ${ }^{31}$ mentre in Dalmazia erano il console britannico Thomas Turner e il poeta Luka Stulić a fargli da "agenti". ${ }^{32}$ Negli ultimi anni della sua vita, poi, Guilford fu assai impegnato nella ricerca di manuali, dizionari e grammatiche per i giovani allievi della sua Accademia; e quando i libri mancavano, il nostro Lord li faceva scrivere ex novo. Ne è un esempio un voluminoso manoscritto in fogli sciolti contenente una Grammatica pratica della lingua Illirica oggi conservato presso l'Archivio della contea di Kent, in Inghilterra. ${ }^{33}$ Come per la copia londinese del Vocabolario di Tanzlingher, anche in questo caso si tratta di un volume redatto in due lingue, italiano e croato. Le caratteristiche formali del manoscritto inducono a pensare che si tratti di una bozza per un manuale destinato agli studenti dell'Accademia Ionica: tutte le pagine hanno infatti una dimensione standard (formato protocollo), sono scritte accuratamente e organizzate in modo omogeneo, divise in due colonne, in modo da avere il testo italiano sulla metà sinistra del foglio e la traduzione croata sulla metà di destra. ${ }^{34} \mathrm{~A}$ suggerire la finalità pratica del volume è anche la presenza di

${ }^{30}$ Oltre ad informare Guilford del fallimento e della conseguente vendita all'asta della stamperia universitaria di Pest gestita da Dimitrije Davidović, Kopitar consigliava all'amico di impiegare «le caractère écclesiastique, comme le seul en usage, d'après les premières éditions du XV siècle, faites a Venise» (M. PARTRIDGE, Jernej Kopitar and Frederick North, 5th Earl of Guilford, cit., p. 282). Il coinvolgimento di Solarić è invece testimoniato da alcuni documenti conservati presso l'Archivio dell'Accademia delle Scienze e delle Arti serba a Sremski Karlovci. Tali documenti saranno oggetto di studio in una prossima pubblicazione.

${ }^{31}$ M. PARTRIDGE, Jernej Kopitar and Frederick North, 5th Earl of Guilford, cit., p. 280.

${ }^{32}$ In una missiva indirizzata a Guilford e datata 21 Aprile 1821, Stulić fa riferimento a dei «Manoscritti illirici» e ad una «Cassettina con li manoscritti greci» (M. FrAJND, Luka Stulić i Lord Nort - prilozi poznavanju veza Dubrovnika i Engleske u XIX veku, cit., p. 108-109).

${ }^{33}$ Il manoscritto fa parte del fondo denominato "The North Papers", che raccoglie il carteggio privato della famiglia. Cfr. M. PARTRIDGe, The First Practical Grammar and Reader of Illyrian, cit., p. 497.

${ }^{34}$ Monica Partridge, che ha scoperto e studiato il manoscritto, sostiene che esso fosse chiaramente pensato per un'eventuale pubblicazione («evidently intended for eventual 
brani destinati alla lettura, perlopiù dialoghi («rasgovori») fra due persone, redatti in lingua "popolare". ${ }^{35}$

Omogeneità, ordine e praticità sembrano essere le direttive seguite anche dal creatore della copia londinese del Vocabolario di Tanzlingher. Anche in questo caso, infatti, si tratta di un bel manoscritto pulito, stilato con attenzione e cura, in una grafia uniforme. I lemmi sono organizzati in colonne, due per ciascuna pagina, che contano fra le 49 e le 60 righe. ${ }^{36}$ A livello di formato, poi, la copia di Londra si presenta come molto più compatta di quella di Padova, costituita da due grandi volumi in folio, pur contenendo quasi lo stesso numero di termini italiani e croati.

Alla luce di quanto detto, appare plausibile che il manoscritto di Londra del Vocabolario di Tanzlingher sia in realtà la bozza di una pubblicazione destinata agli studenti dell'Accademia Ionica, ma mai realizzata. Assieme alla Grammatica manoscritta conservata nell'Archivio della contea di Kent, esso avrebbe costituito uno strumento ideale per chiunque avesse voluto intraprendere lo studio della lingua "illirica": un manuale di grammatica e un dizionario, il perfetto kit di partenza per chi si appresta a studiare una lingua straniera.

Una seconda ipotesi, se vogliamo più semplice, prevede invece che la copia londinese del Vocabolario sia stata prodotta per rispondere alle esigenze private dello stesso Guilford, il quale, come abbiamo detto, stava a sua volta studiando la lingua "illirica".

Chiaramente, le due ipotesi qui presentate necessitano di ulteriori ricerche e verifiche. In primo luogo, un confronto grafologico tra la copia di Londra e alcuni documenti manoscritti, attribuiti ad Ivan Tanzlingher e conservati a Zara, potrebbe chiarire se il manoscritto della British Library sia stato effettivamente compilato dal canonico zaratino, benché sembri poco probabile. In secondo luogo, l'analisi della carta e delle filigrane fornirebbe dati più attendibili in merito alla data di compilazione del manoscritto. Infine, è plausibile che gli archivi croati, serbi e inglesi conservino ulteriori, preziosi documenti che potrebbero aiutare a chiarire la questione.

publication [...] for the use of foreigners with a knowledge of Italian»). La studiosa inglese descrive la lingua del manoscritto come «Illiryan as spoken then by the Slav people living in Dubrovnik» (M. PARTRIDGe, The First Practical Grammar and Reader of Illyrian, cit., pp. 498, 500).

${ }^{35}$ Monica Partridge descrive questa lingua come «the colloquial, non-literary language as heard spoken live in Dubrovnik by the writer» (M. PARTRIDge, The First Practical Grammar and Reader of Illyrian, cit., p. 501).

${ }^{36}$ V. Bockholt, Z. Mestrović, N. Vajs, Nepoznati dvojezični rječnik Ivana Tanclinghera Zanottija, cit., p. 46. 
In generale, e concludendo, appare comunque del tutto lecito affermare che l'eredità di Guilford, la sua attività e i suoi svariati contatti con il mondo slavo meritano di essere ulteriormente investigati anche al di fuori dei confini britannici. Tali ricerche potrebbero infatti gettare nuova luce non solo sulla genesi della copia londinese del Vocabolario di Tanzlingher, ma più in generale sulla circolazione di libri e di manoscritti slavi nella prima metà del XIX secolo.

\author{
Monica Fin
}

OD ZADRA DO BRITISH LIBRARY: ZANIMLJIVO PUTOVANJE REČNIKA TRI PLEMENITA JEZIKA IVANA TANZLINGERA ZANOTIJA

(Rezime)

Usredsređujući se na rekonstrukciju nastanka rukopisa i na odnose filijacije među rukopisima, autorka rada se bavi primerkom Rečnika Ivana Tanzlingera Zanotija koji se čuva u Londonu i iznosi dve radne hipoteze njegovog nastanka: po prvoj bi Tanzlingerov rečnik hrvatskog jezika bio namenjen, u publikovanoj verziji, studentima Jonske akademije čija je ključna figura bio političar i mecena, lord Gilford; po drugoj, rečnik bi, u svojoj londonskoj verziji, bio namenjen privatnoj upotrebi samoga Gilforda koji je i sam učio "ilirski". 